BART PHILLIPS SLOANE

\section{Washington's Headquarters}

Built some years before America's war for independence, the Isaac Potts House at Valley Forge was to serve as General Washington's command post. A score of aides crowded in with him to spend the winter. About eighty percent of the building is original, the rest having been restored according to information obtained through historic research.

Edition limited to 850 offset lithographs, signed by the artist.

Image size: $17 \times 23-1 / 2$

$\$ 70.00$

Framed $\$ 172.00$
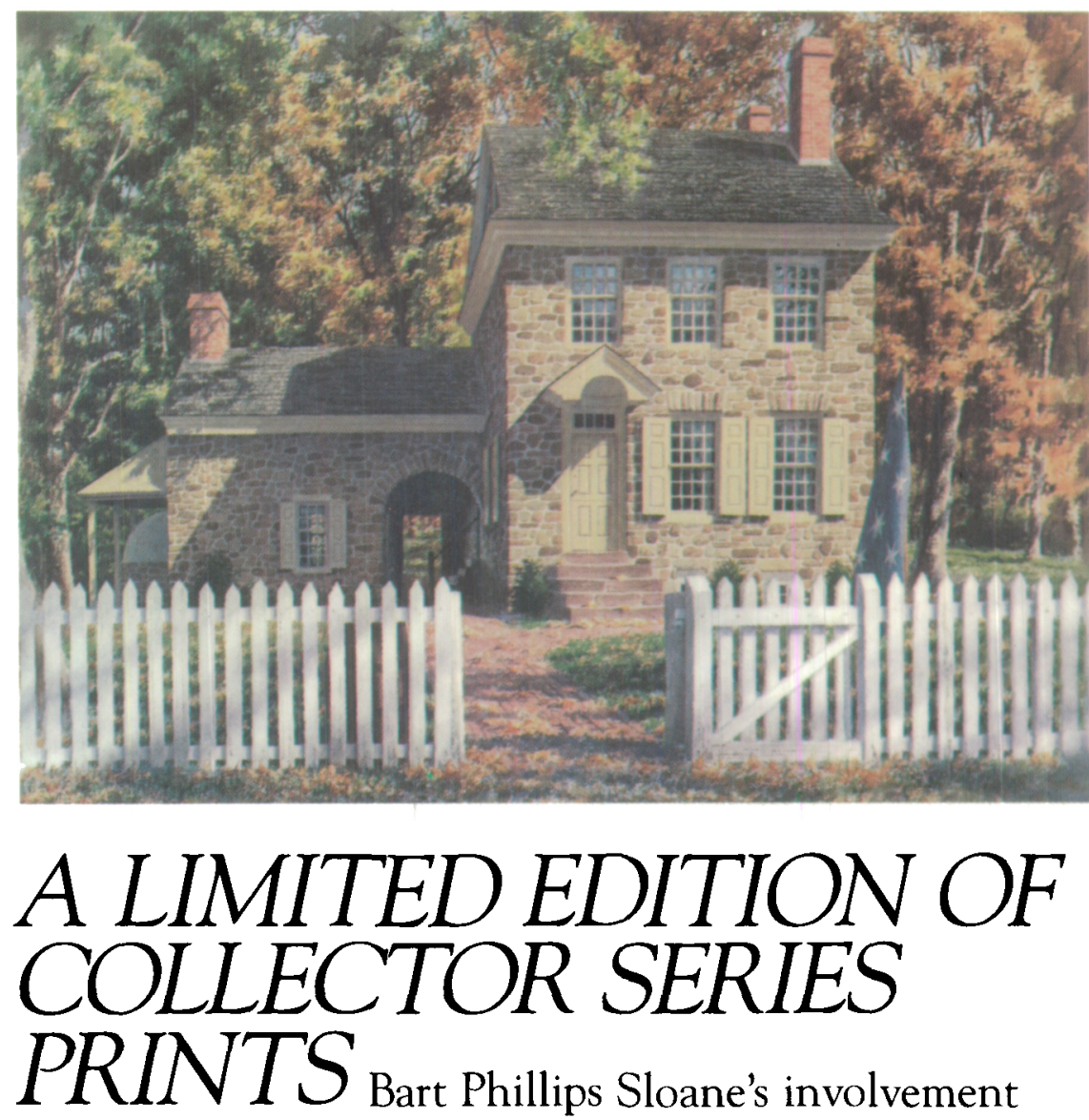
with the graphic arts has found its happiest expression through the medium of watercolor. The qualities of spontaneity, informality and warmth seem quite naturally related to the watercolor. His success with this is attested to not only by the many awards and prizes he has won, but the many happy owners of his works. In Bart's words, "To meet an enthusiastic owner years after the sale is one of my greatest satisfactions."

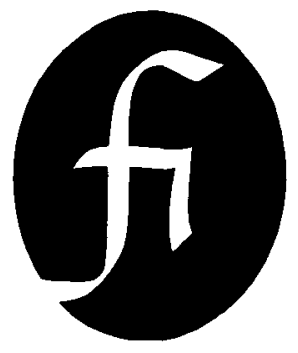

FIRST IMPRESSIONS 6900 Grove Road
Please send me paid. Unframed.

Please send me paid. Framed.

Name

Address

City State Zip print(s) of Washington's Headquarters (a) $\$ 70.00^{*}$ postage print(s) of Washington's Headquarters @ $\$ 172.00^{*}$ postage 


\section{Hamals \\ that serve deserve \\ the best}

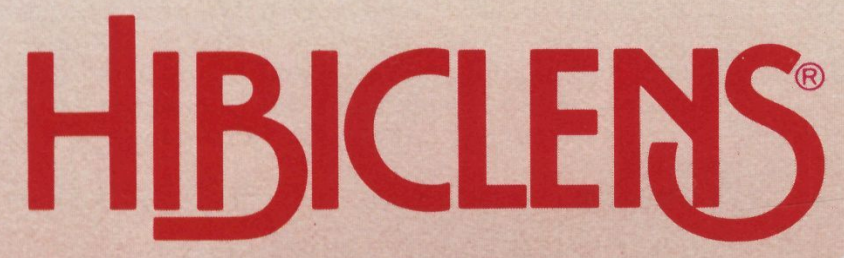

\section{Antiseptic/antimicrobial skin cleanser}

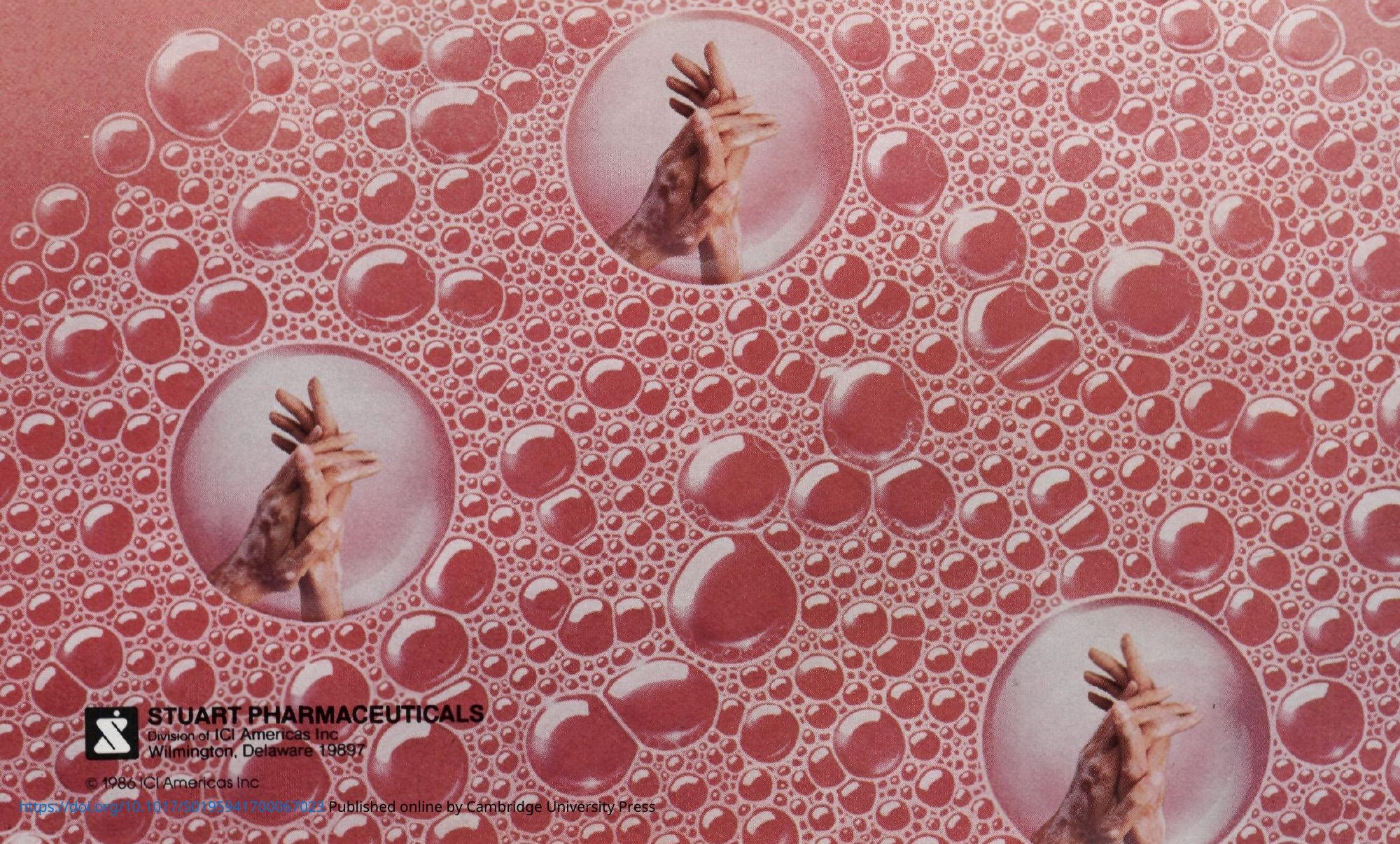

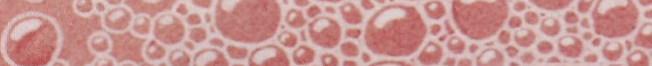

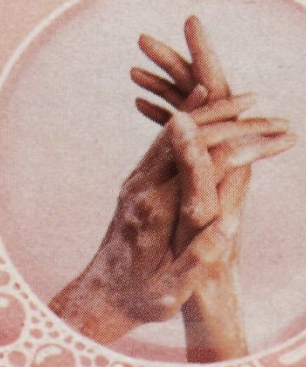

